\title{
Control Strategies for an Automotive Suspension with a MR Damper
}

\author{
Jorge de Jesus Lozoya-Santos * Ruben Morales-Menendez* \\ Juan C. Tudón-Martínez* Olivier Sename ${ }^{* *}$ Luc Dugard $^{* *}$ \\ Ricardo Ramirez-Mendoza* \\ * Tecnologico de Monterrey, Campus Monterrey, CP 64849 Mexico \\ e-mail: \{jorge.lozoya,rmm, A00287r56, ricardo.ramirez\}@itesm.mx \\ ** GIPSA-Lab, Département Automatique, CNRS-Grenoble INP \\ ENSE3, BP 46, F-38402 St Martin d'Hères cedex, France \\ e-mail: \{olivier.sename, luc.dugard\}@gipsa-lab.grenoble-inp.fr
}

\begin{abstract}
A semi-active control system of an automotive suspension with an MagnetoRheological $(M R)$ damper as a key element is considered. Given its hysteretical and nonlinear behavior, the inclusion of a $M R$ damper model in a controller synthesis is presented. Two controllers are proposed from different approaches: $L P V$ control and a Frequency EstimationBased $(F E B)$ control. The $L P V$ controller uses a $L P V$ model of a quarter of vehicle based on the $M R$ damper dynamics. The FEB controller is a model-free controller. These controllers are compared under comfort oriented standards. Simulation results show these controllers as new alternatives with excellent response for comfort and road holding (improving the comfort between 10-20). \%.
\end{abstract}

Keywords: modelling, semi-active, LPV, antiwindup, MR damper, control structure

\section{INTRODUCTION}

The Magneto-Rheological $(M R)$ damper is a non-linear component with dissipative capability used in Automotive Suspension Control System (ASCS), where the damping coefficient varies according to the applied electric current. This damper allows to achieve good comfort, to emphasize road holding and to keep a safety suspension deflection. The approaches in the $A S C S$ can be classified as (a) those with experimental validation and (b) techniques that are not yet practically validated. In the practical side, the model-free controllers Sky-Hook technique, Bolandhemmat et al. [2009] and the Mix-1-sensor strategy, Savaresi and Spelta [2009] are the more efficient for comfort. Also the nonlinear control techniques such as model predictive control and sliding mode control, Dong et al. [2010] and the human simulated intelligent controller, Yu et al. [2008], offers interesting experimental results where comfort and road holding are the objectives but with opportunity areas. In simulation robust solutions has been proposed that seems to be adequate for implementation in real time systems, for instance the $\mathrm{H}_{\infty}$ technique, Choi and Sung [2008], using a linear $M R$ damper model and the nonlinear control based on linear parameter varying $/ \mathrm{H}_{\infty}$, Do et al. [2010]. The aforementioned control strategies in literature shows that:

(1) The controller output is not the damper manipulation (with Ampere units), commonly the desired force of the $M R$ damper or the damping coefficient are computed. These designs compromise the controller

\footnotetext{
* Thanks to the CNRS, France and CONACyT 06/07, Mexico for the support under the Postgraduate Cooperation Project 2007-2011.
}

practical feasibility by adding a mapping algorithm from control output to manipulation units and may deteriorate the performances.

(2) In experimental evaluations, the model-free strategies are efficient and feasible implementations when they are compared with more complex controllers.

(3) The anti-windup mechanism is assumed by applying inverse $M R$ damper models; however the controllers have not a feedback of a windup effect. Therefore the mechanism is not a reaction but an assumption.

Hence, the development of feasible and multi-objective controllers that overcome the above limitations is an opportunity area. This paper deals with: (a) the design of a $L P V$ controller with one scheduling parameter based on a simple non-linear $M R$ damper model, (b) the design of a model-free controller based on the deflection frequency estimation and (c) a comparison of these controllers under the BS 6841 comfort oriented standard, Griffin [1996]. The controllers evaluation uses a vertical nonlinear Quarter of Vehicle, $(Q \circ V)$ where the $M R$ damper model includes the hysteresis simulation. This work is an extension of the work proposed by Do et al. [2010] and Lozoya-Santos et al. [2010b]. This paper is organized as follows. Section 2 specifies the semi-active control strategies. Section 3 presents the nonlinear $Q_{o} V$ model and the simulation results. The discussion of results are on section 4 . Section 5 concludes the paper. Table 1 presents the paper nomenclature.

\section{SEMI-ACTIVE CONTROL STRATEGIES}

Two controllers are proposed: an $L P V$-based and a Frequency-Estimation-Based. 


\section{$2.1 L P V$-based controller}

This control strategy applies the $H_{\infty}$ control design for polytopic systems, Poussot-Vassal et al. [2008], Do et al. [2010], using a new $L P V$ model for a $Q o V$, LozoyaSantos et al. [2010b], which allows to have one scheduling parameter.

The representation of a $Q o V$ in the $L P V$ framework by including a $M R$ damper in the suspension and considering $F_{d z}=0$ and doing $z=z_{s}-z_{u s}$ can be defined as:

$\left[\begin{array}{c}\dot{z}_{s} \\ \ddot{z}_{s} \\ \dot{z}_{u s} \\ \ddot{z}_{u s}\end{array}\right]=A_{s} z_{s u s}+B_{s} \cdot u_{c}+B_{s 1} \cdot z_{r}, y=C_{s} z_{s u s}+D\left[\begin{array}{c}u_{c} \\ z_{r} \\ 0\end{array}\right]$

where,

$$
\begin{aligned}
& A_{s}=\left[\begin{array}{cccc}
0 & 1 & 0 & 0 \\
\frac{-k_{s}-k_{p}}{m_{s}} & -\frac{c_{p}}{m_{s}} & \frac{k_{s}+k_{p}}{m_{s}} & \frac{c_{p}}{m_{s}} \\
0 & 0 & 0 & 1 \\
\frac{k_{s}+k_{p}}{m_{u s}} & \frac{c_{p}}{m_{u s}} & \frac{-k_{s}-k_{p}-k_{t}}{m_{u s}} & -\frac{c_{p}}{m_{u s}}
\end{array}\right], \\
& z_{\text {sus }}=\left[\begin{array}{c}
z_{s} \\
\dot{z}_{s} \\
z_{u s} \\
\dot{z}_{u s}
\end{array}\right], B_{s}=\left[\begin{array}{c}
0 \\
-\frac{c_{M R} \cdot \rho_{s a t} \rho_{s a}}{m_{s}} \\
0 \\
\frac{c_{M R} \cdot \rho_{s a t} \rho_{s a}}{m_{u s}}
\end{array}\right] \\
& B_{s 1}=\left[\begin{array}{c}
0 \\
0 \\
0 \\
\frac{k_{t}}{m_{u s}}
\end{array}\right], C_{s}=\left[\begin{array}{c}
0 \\
1 \\
0 \\
-1
\end{array}\right], D=0, u_{c}=I
\end{aligned}
$$

According to system (1), the $M R$ damping force is described as:

$$
f_{M R}=c_{M R} \cdot u_{s a} \cdot \rho_{s a t}+c_{p} \dot{z}+k_{p} z,
$$

where $u_{s a}=u_{c} \cdot \rho_{s a}$ is the exogenous input of the system (1) and $\rho_{s a}$ defines the semi-activeness of $u_{c}$, i. e. if $u_{c}$ can be introduced to the control system or if it is canceled according to,

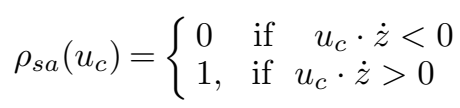

The saturation and nonlinearities of the $M R$ damper are coped with the parameters:

$$
\begin{gathered}
\rho_{\text {sat }}=\left[\frac{\tanh \left[\left(u_{s a}\right) /\left(I_{\max } \cdot \rho\right)\right]}{\left(u_{s a}\right) /\left(I_{\max } \cdot \rho\right)}\right], \quad \rho_{\text {sat }} \in[0,1] \\
\rho=\frac{|\dot{z}|}{\dot{z}_{\infty}}, \quad \rho \in[0,1]
\end{gathered}
$$

The model (2), Lozoya-Santos et al. [2010b], has a simple structure, it allows to quantify the linear effect of the current on the damping coefficient, and it simulates the damping variability, three important characteristics for

\begin{tabular}{|c|c|}
\hline Variable & Description \\
\hline$c_{M R}$ & $\begin{array}{l}\text { Maximum damping coefficient in } M R \text { damper } \\
\text { due to I }((N \cdot A) / m)\end{array}$ \\
\hline$c_{p}$ & Passive damping coefficient $(\mathrm{Ns} / \mathrm{m})$ \\
\hline$f$ & Frequency for weighting function $(\mathrm{Hz})$ \\
\hline$\hat{f}$ & Estimated frequency by $F E B$ controller $(\mathrm{Hz})$ \\
\hline$f_{f r}$ & friction damping force $(N)$ \\
\hline$f_{M R}$ & $M R$ damping observed force $(N)$ \\
\hline$f_{\text {steering }}$ & Force generated for a steering action $(\mathrm{N})$ \\
\hline$h_{p 1}, h_{p 2}$ & Coefficients for the hysteresis, \\
\hline$i$ & The $i^{t h}$ sample of the deflection velocity \\
\hline$k_{p}$ & Passive stiffness coefficient $(\mathrm{N} / \mathrm{m})$ \\
\hline$k_{s}$ & Stiffness in a quarter of vehicle suspension $(\mathrm{N} / \mathrm{m})$ \\
\hline$k$ & Receding horizon in order to compute $\|\dot{z}\|_{\infty}$ \\
\hline$m_{M R d}$ & Virtual mass of $M R$ damper \\
\hline$m_{s}$ & Sprung mass of a quarter of vehicle $(K g)$ \\
\hline$m_{u s}$ & Unsprung mass of a quarter of vehicle $(K g)$ \\
\hline$n$ & $\begin{array}{l}\text { Subscript for } H_{\infty} \text { performance objectives } \\
n=\left\{\ddot{z}_{s}, z_{u s}\right\}\end{array}$ \\
\hline$t$ & Time (s) \\
\hline$\overline{u_{s a}}$ & $\begin{array}{l}\text { Determines the damping coefficient magnitude } \\
\text { when the device is in tension or compression. }\end{array}$ \\
\hline$w$ & Weighted $z_{r}(\mathrm{~m})$ \\
\hline$y_{1}, y_{2}$ & Coefficients for the $M R$ sigmoid behavior \\
\hline$z, z_{\text {def }}$ & Piston deflection $(\mathrm{m})$ \\
\hline$z_{r}$ & Road profile shaped as chirp sinusoidal \\
\hline$\dot{z}, \dot{z}_{\text {def }}$ & Piston deflection velocity $(\mathrm{m} / \mathrm{s})$ \\
\hline$\dot{z}_{\min }$ & Minimum measured $\dot{z}$ \\
\hline$\dot{z}_{\max }$ & Maximum measured $\dot{z}$ \\
\hline$\ddot{z}_{w}$ & Controlled $\ddot{z}\left(\mathrm{~m} / \mathrm{s}^{2}\right)$ \\
\hline$\dot{z}_{s}$ & Sprung mass velocity $(\mathrm{m} / \mathrm{s})$ \\
\hline$\ddot{z}_{s}$ & Sprung mass acceleration $\left(\mathrm{m} / \mathrm{s}^{2}\right)$ \\
\hline$z_{u s}$ & Unsprung mass displacement $(\mathrm{m})$ \\
\hline$z_{u s w}$ & Controlled $z_{u s}(\mathrm{~m})$ \\
\hline$\dot{z}_{u s}$ & Unsprung mass velocity $(\mathrm{m} / \mathrm{s})$ \\
\hline$\ddot{z}_{u s}$ & Unsprung mass acceleration $\left(\mathrm{m} / \mathrm{s}^{2}\right)$ \\
\hline$\overline{B W_{j}}$ & j-esime BandWidth in FEB control \\
\hline$I$ & Applied current (A) \\
\hline$I_{\max }$ & Maximum current allowed by the $M R$ damper \\
\hline$R$ & Sinusoidal amplitude $(\mathrm{m})$ \\
\hline$W_{z_{r}}$ & Weighting function for road profile \\
\hline$W_{\ddot{z}_{s}}$ & $\begin{array}{l}\text { Weighting function for sprung } \\
\text { mass acceleration }\end{array}$ \\
\hline$W_{z_{u s}}$ & $\begin{array}{l}\text { Weighting function for unsprung } \\
\text { mass displacement }\end{array}$ \\
\hline$\left|\|\dot{z} \mid\|_{\infty}\right.$ & $\begin{array}{l}\text { Absolute maximum deflection velocity, } \\
\dot{z}_{\infty}:\left\{\left\|\left(\left|\dot{z}_{i}\right|\right)\right\|_{\infty_{i-k}} \in\left[\dot{z}_{\min }, \dot{z}_{\max }\right]\right\}\end{array}$ \\
\hline$\rho$ & $\begin{array}{l}\text { Effect of the mechanical and hydraulic properties } \\
\text { of the damper on } c_{M R}\end{array}$ \\
\hline$\rho_{\text {sa }}$ & $\begin{array}{l}\text { Defines the semi-activeness of the LPV controller } \\
\text { output }\end{array}$ \\
\hline$\rho_{\text {sat }}$ & $\begin{array}{l}\text { Dynamic saturation of the electric current } \\
\text { limited to a maximum current value } I_{\max } \text { and } \rho\end{array}$ \\
\hline$\rho^{*}$ & Scheduling parameter for LPV controller \\
\hline$\omega$ & Displacement frequency $(\mathrm{rad} / \mathrm{s})$ \\
\hline$\zeta$ & Damping coefficient in weighting function \\
\hline$\zeta_{M R}$ & $\begin{array}{l}\text { Varying damping coefficient in } M R \text { damper } \\
\text { due to current } I((N) / m)\end{array}$ \\
\hline
\end{tabular}
controller synthesis.

The dissipativity specifies the damping coefficient must be positive. The term of equation (2) can be expressed as:

$$
f_{M R}(I)=c_{M R} \cdot I \cdot \operatorname{sign}(\dot{z}) \cdot \rho_{\text {sat }}
$$

Table 1. Nomenclature

By substituting (3), (4), (5), in (6), and assuming semiactiveness, $\rho_{s a}=1$,

$$
f_{M R}(I)=c_{M R} \cdot I_{\max } \cdot \frac{\dot{z}}{\dot{z}_{\infty}} \cdot \tanh \left[\frac{I}{I_{\max } \cdot \rho}\right]
$$

Finally dividing between $\dot{z}$, the damping coefficient due to $I$ is, 
Table 2. Weighting functions

\begin{tabular}{|c|c|c|c|c|}
\hline$W$ & Objective & $K_{n}$ & $\tau_{n}$ & $\zeta$ \\
\hline$W_{\ddot{z}_{s}}$ & Sprung mass acceleration & 0.8 & 0.008 & 0.7 \\
$W_{z_{u s}}$ & Unsprung mass displacement & 100 & 0.0145 & 0.4 \\
$W_{z_{r}}$ & Road profile & 0.07 & 0 & 0 \\
\hline
\end{tabular}

$\zeta_{M R}(I)=\frac{f_{M R}(I)}{\dot{z}}=\frac{c_{M R}}{\dot{z}_{\infty}} \cdot I_{\max } \cdot \tanh \left[\frac{I}{I_{\max } \cdot \rho}\right]$

where $\zeta_{M R}(I)$ is the $M R$ damping coefficient due to the electric current changes. Since $\operatorname{sign}\left(f_{M R}(I)\right)=\operatorname{sign}(\dot{z})$, it can be concluded that:

(1) $\zeta_{M R}$ is always positive and proportional to $I$.

(2) $I$ is bounded by the scheduling parameter $\rho$ to the value $I_{\max }$.

Therefore the $L P V$ controller varies the damping of the semi-active suspension.

Finally by the semi-activeness and dissipativiy constraints of the system (1), the chosen scheduling parameter is:

$$
\rho^{*}=\rho_{s a} \cdot \rho_{\text {sat }} \in[0,1]
$$

The generalized system for the $H_{\infty} / L P V$ controller synthesis with one scheduling parameter is done according to Do et al. [2010], where a filter is added to the input of (1) in order to be proper for the $L P V$ synthesis. Figure 1 shows the obtained $Q o V$ structure by using the $M R$ damper model (2) and an ideal linear design. With the new

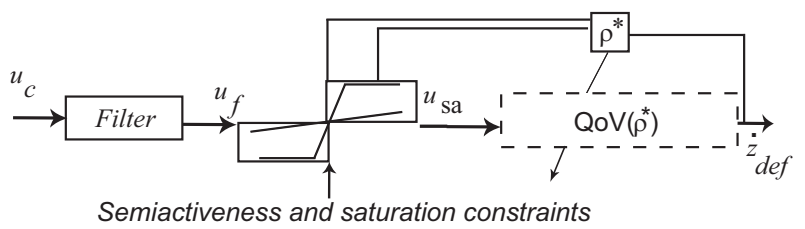

Fig. 1. Model with a semi-active bounded input saturation.

structure, the states transition matrix $A$ used for controller synthesis contains $\rho^{*}$ according to Do et al. [2010],

$$
A\left(\rho^{*}\right)=\left[\begin{array}{cc}
A_{s} & \rho^{*} B_{s} C_{f} \\
0 & A_{f}
\end{array}\right],\left(\begin{array}{l}
\dot{x}_{f} \\
u_{f}
\end{array}\right)=\left[\begin{array}{cc}
A_{f} & B_{f} \\
C_{f} \rho^{*} & 0
\end{array}\right]\left(\begin{array}{l}
x_{f} \\
u_{c}
\end{array}\right)
$$

where $\left(u_{f}\right)$ is the filtered controller output, and $A_{f}, B_{f}$, and $C_{f}$ are the filter state space representation. In order to meet the control specifications, three $H_{\infty}$ weighting functions were defined by a a priori knowledge according to Table 2 and equation 11. Figure 2 shows the control strategy structure, with:

$$
W=\frac{K_{n}}{\tau_{n}^{2} \cdot s^{2}+2 \tau_{n} \zeta_{n} \cdot s+1}
$$

\subsection{Frequency Estimation-Based Controller (FEBC)}

The comfort and road holding conditions depend on the displacement frequency $f_{z_{r}}$ in the suspension of a $Q_{o} V$. In order to meet the specified performances, for a given $f_{z_{r}}$ corresponds a damping coefficient $\zeta$. Hence, by having a measurement or estimation of $f_{z_{r}}$ is possible to assign specific values of $\zeta$ according to a pre-analysis of the frequency responses in the performances objectives.

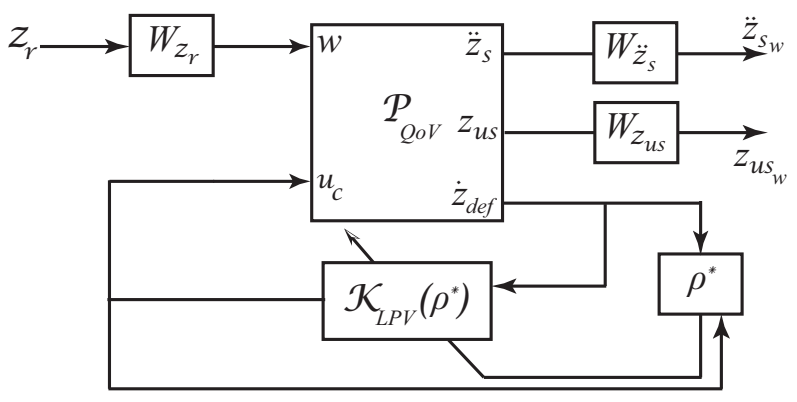

Fig. 2. LPV-based approach for the QoV with semi-active suspension

In a $Q o V$ with a $M R$ suspension, the damping varies according to the applied electric current, $\zeta_{M R}(I)$. Hence, it is possible to hold a specific suspension condition based on a estimated frequency $\hat{f}_{z_{r}}$ and applying an electric current.

By assuming a harmonic motion of the damper piston, two state variables of the system (1) can be described by:

$$
\begin{aligned}
& z \approx R \cdot \sin (\omega \cdot t) \\
& \dot{z} \approx \omega \cdot R \cdot \cos (\omega \cdot t)
\end{aligned}
$$

where the signals $z$ and $\dot{z}$ are measured and there is no feasible prior information of $\omega$. Using these two measurements, it is possible to estimate the road profile frequency and to decide which damping to apply in order to achieve comfort or road holding conditions. By using the root mean square $(\mathrm{rms})$ values of deflection and velocity deflection of the $M R$ damper piston, the estimation of the frequency $\omega$ is given by,

$$
\hat{f}=\left(\dot{z}_{r m s}\right) /\left(2 \cdot \pi \cdot z_{r m s}\right)
$$

By doing a numerical integration to compute the discrete rms values of $\dot{z}_{r m s}$ and $z_{r m s}$,

$$
\hat{f}=\sqrt{\frac{\left(\dot{z}_{1}^{2}+\dot{z}_{2}^{2}+\cdots+\dot{z}_{n}^{2}\right)}{\left(z_{1}^{2}+z_{2}^{2}+\cdots+z_{n}^{2}\right) \cdot 4 \pi^{2}}}
$$

The number of samples $n$ in order to compute the rms values corresponds to $2 \mathrm{~Hz}$ according to the invariant point of the frequency response of the $Q_{o} V$ for the sprung mass, see Cartwright [2007]. Therefore, using a sample time of $1 / 512$, the size of window $n$ is 256 samples. In simulation, the final value of $n$ were optimized to 128 in order to have a wider window in frequency estimation. The manipulation corresponding to a given frequency is obtained observing the frequency response function for the controlled variables, defining a lookup table in order to assure the desired performances for comfort and road holding.

\section{SIMULATION}

The performance of $A S C S$ is evaluated in frequency and time domains. In the frequency domain, the industrial specifications, are defined in the span of $[0-20] \mathrm{Hz}$ as follows:

- Comfort: In the span of [0-20] Hz, the maximum gain of the frequency response $\ddot{z}_{s} / z_{r}$ must be kept as low as possible in order to avoid general sickness, Griffin 
[1996]. The maximum gain of the frequency response $z_{s} / z_{r}$ must be kept below 1.8 in the span [0-5] hz, , Poussot-Vassal et al. [2008].

- Road holding : The frequency response $\left(z_{u s}-z_{r}\right) / z_{r}$ gain ideally must stand closer to $0 \mathrm{~mm}$ (zero), Poussot-Vassal et al. [2008].

- Suspension deflection: A constraint on the deflection of the actuator $z_{d e f} / z_{r}$ is hold between 0 and $20 \mathrm{~Hz}$ in order to preserve the lifetime cycle, Poussot-Vassal et al. [2008].

The pseudo-Bode evaluate the performance quality and the Pseudo Spectral Density (PSD) quantifies the performance improvement as in Do et al. [2010]. In the time domain, interest signals versus time as well as force-velocity plots show the transient responses, and the Root Mean Square $(R M S)$ index the transient performances. The British Standard BS 6841 is a human vibration comfort standard which weights $\ddot{z}_{s}$ through the following transfer function:

$$
W b_{B S 6841}=\frac{s^{3}+118 s^{2}+2001 s+24810}{s^{4}+\frac{1046 s^{3}}{5}+15570 s^{2}+382800 s+6384000}
$$

where the rms spans are: comfortable $\left(r m s_{\ddot{z}_{s}}<0.315\right)$, little uncomfortable $\left(0.315<r m s_{\ddot{z}_{s}}<0.63\right)$, and uncomfortable $\left(r m s_{\ddot{z}_{s}}>0.63\right)$. Given the specifications, two controllers are proposed based on $L P V$ techniques and the estimation of frequency.

The considered $M R$ damper is an $A C D E L C O$ damper; it is a component of the semi-active suspension of a Cadillac vehicle. The experimental data for identification is a sinusoidal displacement with variable amplitude $\mp 8$ $\mathrm{mm}$ and constant frequency of $7 \mathrm{~Hz}$. The amplitude varies randomly each 3 cycles. The electric current is a random walk shape, Ljung [1999], with a span of $0-2.5 \mathrm{~A}$. The spans of $\dot{z}$ and $f_{M R}$ were $\mp 0.6 \mathrm{~m} / \mathrm{s}$ and $\mp 2500 \mathrm{~N}$. The sampling frequency was $512 \mathrm{~Hz}$.

The dynamical equations of a $Q o V$ are governed by:

$$
\begin{aligned}
& m_{s} \ddot{z}_{s}=-k \cdot z-f_{M R}+f_{\text {steering }} \\
& m_{u s} \ddot{z}_{u s}=k \cdot z+f_{M R}-k_{t}\left(z_{u s}-z_{r}\right)
\end{aligned}
$$

taken from a Renault Megane Coupe ${ }^{T M}$ (Poussot-Vassal et al. [2008]). The $f_{\text {steering }}$ is considered zero because the vehicle is considered running in straight line at 40 $\mathrm{Km} / \mathrm{h}$. The nonlinear model of the $Q o V$ with a semi-active suspension is simulated using the model:

$$
\begin{aligned}
f_{M R}= & k_{p} z+c_{p} \dot{z}+m_{M R} \cdot \ddot{z}+f_{f r} \cdot \tanh \left(h_{p 1} \cdot \dot{z}+h_{p 2} \cdot z\right)+ \\
& c_{M R} \cdot I \cdot \tanh \left(y_{1} \cdot \dot{z}+y_{2} \cdot z\right)
\end{aligned}
$$

This $M R$ damper model shows good estimation of the hysteresis phenomenon in the force versus velocity map, Figure 3.
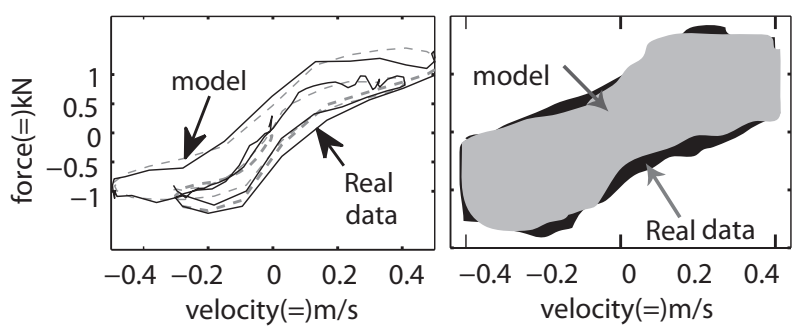

Fig. 3. Force-velocity plane evaluation.

\begin{tabular}{|c|c|c|c|c|c|}
\hline \multicolumn{3}{|c|}{$\begin{array}{l}\text { QoV coefficients for controller } \\
\text { synthesis and simulation }\end{array}$} & \multicolumn{3}{|c|}{$\begin{array}{l}\text { MR damper coefficients } \\
\text { for control synthesis }\end{array}$} \\
\hline Parameter & Value & Units & Parameter & Value & Units \\
\hline$m_{s}$ & 315 & $\mathrm{~kg}$ & $c_{p}$ & 987 & $\mathrm{Ns} / \mathrm{m}$ \\
\hline$m_{u s}$ & 37.5 & $\mathrm{~kg}$ & $k_{p}$ & -10239 & $\mathrm{~N} / \mathrm{m}$ \\
\hline k & 29.5 & $\overline{(\mathrm{kN}) / \mathrm{m}}$ & $\bar{c} c_{M R}$ & 441 & $\mathrm{Ns} /(\mathrm{Am})$ \\
\hline$k_{t}$ & 210 & $(\mathrm{kN}) / \mathrm{m}$ & $k$ & 124 & - \\
\hline \multicolumn{6}{|c|}{ Parameters of the $M R$ damper in non linear $Q o V$ simulation } \\
\hline Parameter & Value & Units & Parameter & Value & Units \\
\hline$f_{f r}$ & 142 & $\mathrm{~N}$ & $h_{p 1}$ & 45 & $\mathrm{~s} / \mathrm{m}$ \\
\hline$h_{p 2}$ & 37 & $1 / \mathrm{m}$ & $c_{p}$ & 245 & $\mathrm{Ns} / \mathrm{m}$ \\
\hline$k_{p}$ & -5982 & $\mathrm{~N} / \mathrm{m}$ & $\begin{array}{c}c_{M R} \\
\end{array}$ & 444 & $\mathrm{Ns} /(\mathrm{Am})$ \\
\hline$m_{M R d}$ & 7 & $\mathrm{~kg}$ & $y_{1}$ & 7.89 & $\mathrm{~s} / \mathrm{m}$ \\
\hline$y_{2}$ & -13.8 & $1 / \mathrm{m}$ & - & - & - \\
\hline
\end{tabular}

The parameter values for the nonlinear simulation of the $Q o V$ as well as the parameter values for the $L P V \quad Q o V$ used in controller synthesis are shown in Table 3.

Table 3. Parameter values

The road profile in the $Q o V$ simulations was sinusoidal displacement with constant amplitude of $15 \mathrm{~mm}$ and incremental frequency between 0.1 and $20 \mathrm{~Hz}$ each three cycles.

In order to define the comfort and road holding conditions in the frequency domain, a set of three open loop simulations with electrical current of $I=0,1.25,2.5 \mathrm{~A}$ was done. The obtained pseudo-Bodes are shown in Fig. 4. The simulation with a constant electric current $I=1.25 \mathrm{~A}$ is considered the passive suspension.

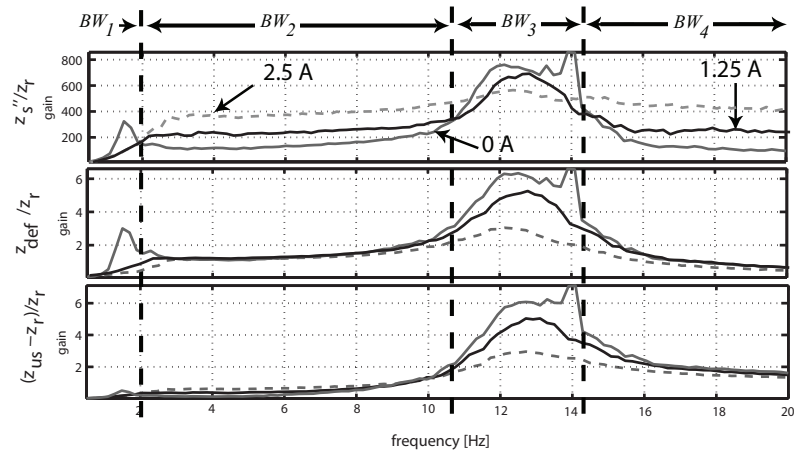

Fig. 4. Pseudobodes for open loop transfer functions: $\ddot{z}_{s} / z_{r}, z_{\text {def }} / z_{r}$, and $\left(z_{u s}-z_{r}\right) / z_{r}$. The plots show the allowed gain variation when the current is $0 \mathrm{~A}, 1.25$ and $2.5 \mathrm{~A}$.

The comfort condition is achieved in $\ddot{z}_{s} / z_{r}$, Fig. 4 top plot, for frequencies between 0 and $2 \mathrm{~Hz}$, called BandWidth 1 $\left(B W_{1}\right)$, a comfort condition and good handling is achieved with current $I=2.5 \mathrm{~A}$ in order to limit the gain below 200. A current $I=0 \mathrm{~A}$, in the range from $2-10.5 \mathrm{~Hz}$, called $B W_{2}$, and from $14-20 \mathrm{~Hz}$, called $B W_{4}$, must actuate over the damper in order to kept the comfort condition. In $B W_{3}$ with a span of $10.5-14 \mathrm{~Hz}$, due to the tire-hop frequency, the applied current must be $I=2.5 \mathrm{~A}$ in order to decrease the gain in $\ddot{z}$.

The suspension deflection transfer function $z_{s}-z_{u s}$ is improved by holding $I=2.5$ A below $3 \mathrm{~Hz}$ and between 11-15 Hz. In the span from $3-11 \mathrm{~Hz}$ and $15-20 \mathrm{~Hz}$, the applied current has not influence on this objective, see Fig. 4 middle plot.

Regarding to the road holding oriented transfer functions $z_{u s}-z_{r} / z_{r}$, in frequencies $0-2 \mathrm{~Hz}, 2.5 \mathrm{~A}$ meet the industrial 
specifications, sharing this feature with comfort. From 2-8 $\mathrm{Hz}, 0 \mathrm{~A}$ keeps low the gain, close to zero, hence comfort condition shares these current values in later frequency spans. A difference from comfort, over $8 \mathrm{hz} 2.5 \mathrm{~A}$ is desirable to allow the road holding, see Fig. 4 bottom plot.

Given the last comments on ideal currents in the frequency bandwidth for comfort and road holding, the lookup Table 4 summarizes the best case current profile in order to accomplish with comfort and road holding.

Table 4. Lookup table for the best performance in comfort and road holding

\begin{tabular}{|c|c|c|c|c|}
\hline$\hat{f}(=) h z$ & $0-2$ & $2-10.5$ & $110.5-14$ & $14-20$ \\
\hline$I(=) A$ & 2.5 & 0 & 2.5 & 0 \\
\hline
\end{tabular}

The automatic control strategies based on LPV and FEB controllers were simulated and compared with passive suspension system. Figures 5 and 6 shows the PseudoBodes and the percentage of $P S D$ improvement in the specified bandwidths.

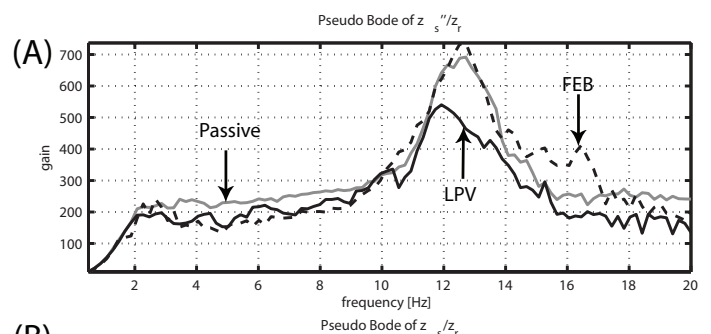

(B)

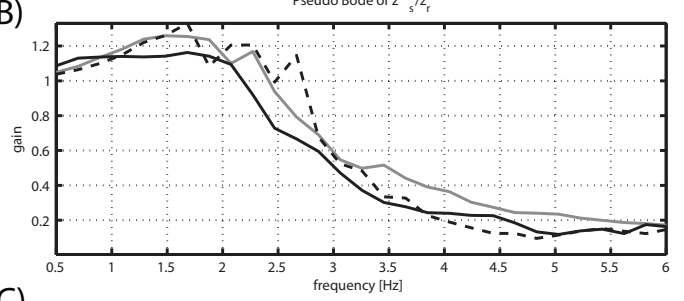

(C)

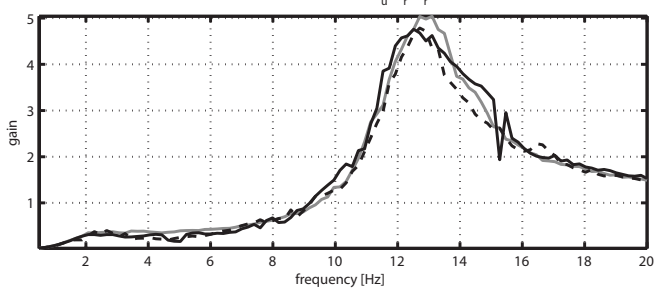

Fig. 5. Pseudo-Bodes for transfer functions: (A) $\ddot{z}_{s} / z_{r}$, (B) $z_{s} / z_{r},(\mathrm{C}) z_{d e f} / z_{r}$, and (D) $\left(z_{u s}-z_{r}\right) / z_{r}$.

A bump is applied in order to evaluate the regulatory transient response of the control systems. The bump has an amplitude of $160 \mathrm{~mm}$ and a frequency of $0.2 \mathrm{~Hz}$. Only the first crest is used as bump. Figure 7 shows the transient responses and Table 5 presents the rms values.

Table 5. Rms results for regulatory transient response.

\begin{tabular}{|c||c|c|c|}
\hline Variable & LPV & FEB & Passive \\
\hline$r m s_{\ddot{z}_{s}}$ & 0.3208 & 0.255 & 0.2914 \\
\hline$r m s_{z_{\text {def }}}$ & 0.003 & 0.0046 & 0.0029 \\
\hline$r m s_{z_{u s}-z_{r}}$ & $9 \mathrm{e}-004$ & $8.1 \mathrm{e}-004$ & $8.3 \mathrm{e}-004$ \\
\hline
\end{tabular}
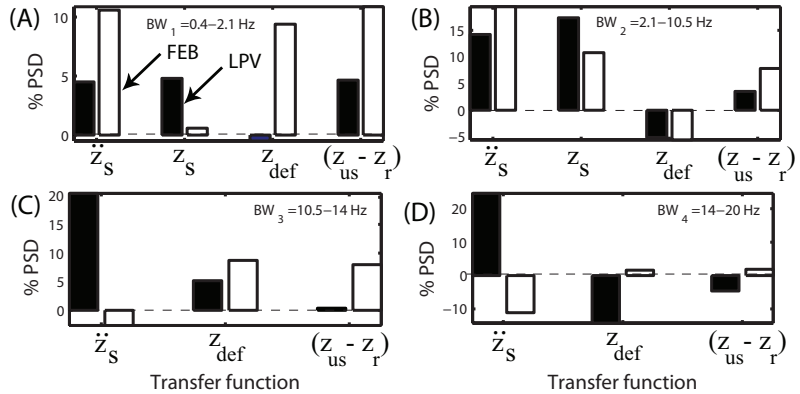

Fig. 6. Percentage of PSD improvements for (A) $B W_{1}$, (B) $B W_{2}$, (C) $B W_{3}$, and (D) $B W_{4}$. Horizontal axis show the improvements in $\ddot{z}_{s}, z_{s}, z_{\text {def }}$, and $z_{u s}-z_{r}$. Vertical axis show PSD $=\left(P S D_{\text {passive }}-\right.$ $\left.P S D_{\text {controller }}\right) / P S D_{\text {passive }}$, hence for positive values of the percentage of PSD, the $A S C S$ is better than passive suspension.
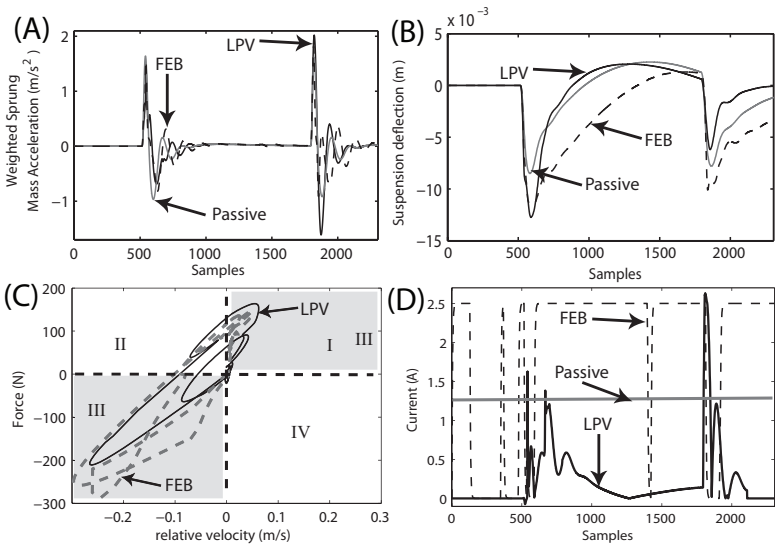

Fig. 7. Transient response for $\mathrm{QoV}$ with the different controllers: (A) Sprung mass acceleration, (B) Suspension deflection, (C) force-velocity in MR damper, and (D) the manipulation.

\section{DISCUSSION OF RESULTS}

By the side of frequency domain, in comfort performance, the $L P V$-based and the FEB-based controllers have better performances than passive one in $[0-10] \mathrm{Hz}$, Figures 5 (A) and 5 (B). The $L P V$-based controller overpasses the FEB controller in the transfer function $\ddot{z}_{s} / z_{r}$ in $B W_{3}$ and $B W_{4}$. For road holding the control strategies present a similar performance than the passive suspension, Figure 5 (C). Both controllers equal the suspension deflection in passive suspension in $B W_{3}$. The quantitative index are given by the percentage of PSD improvement, in comfort both controllers improve between 10-15\% the passive suspension in $B W_{1}$ and $B W_{2}$, Fig. $6(\mathrm{~A})$ and (B). The LPV-Based controller reaches a $20 \%$ in $B W_{3}$ and $B W_{4}$ while FEB-based controller is not better than the passive one, Fig. 6 (C) and (D). In suspension deflection, the improvement is between $5-10 \%$ in $B W_{3}$ for $z_{\text {def }}$, although in $B W_{2}$ and $B W_{4}$ the passive suspension is better. Finally, the road holding condition is improved in all the bandwidths with a span of 5-10\%. In general, both controllers improved the comfort condition without affecting the other performances. 
Regarding to the transient response, for the sprung mass acceleration, the FEB-based controller shows the minimum peak-to-peak acceleration in both, rebound and compression, although the $L P V$-based controller offers a better decay ratio, Figure 7 (A). In suspension deflection, the $L P V$-based controller achieves a safe and soft deflection response (dynamic damping) while the FEB-based controller presents a hard damping. LPV control overpasses both, passive and FEB-based suspensions, Figure 7 (B). Observing the semi-activeness, Figure 7 (C) shows the dissipated forces by the controllers. $f_{M R}$ and $\dot{z}_{d e f}$ remain mainly in the quadrants I and III, shadowed in figure, called semi-active quadrants. The hysteresis is present in the $M R$ damper simulation passing through quadrant IV. Finally the FEB-based controller, by design, only shows two damping coefficients, related to the electric current levels: 0 and $2.5 \mathrm{~A}$, for low and high damping. Hence, their design has not the problem of a saturated manipulation. By other side, the $L P V$ controller, includes the saturation in the scheduling parameter, but it applies the current in a more efficient manner, Figure 7 (D). Table 5 indicates that the rms of weighted $\ddot{z} s$ delivered by FEB-based controller accomplishes with a comfortable vibration while the LPVbased and passive are in the limit to be considered a little uncomfortable, according to BS 6841 classification. This high comfort performance of FEB allows higher suspension deflections while it maintains the road holding.

The simulation results show that the $L P V$ and $F E B$ control strategies have the following characteristics:

(1) The controllers output is the electric current to apply through $M R$ damper coil.

(2) The controllers achieve the objectives with a bounded output.

(3) The scheduling parameter in the LPV controller is based only in one measurement: the velocity deflection. When no velocity sensor is present, this signal can be estimated through numerical computation of suspension acceleration or deflection.

(4) There are no computing time restrictions, hence the controllers allows sampling time of $512 \mathrm{hz}$.

(5) The controllers are once-time computed off-line and achieve the objective performances on-line.

\section{CONCLUSION}

Two controllers for automotive suspensions with $M R$ dampers are proposed: one model-based and one modelfree. The LPV-based controller bases its design on one scheduling parameter and one measurement taking into account the saturation input, semi-activeness and dissipativity constraint of the $M R$ damper. This is achieved by applying a simple nonlinear MR damper model. The model-free controller named FEB uses two measurements to estimate the road profile frequency and to decide which damping to apply to accomplish with requirements.

In the literature the common approach for the controller output is the $M R$ damper force adding the necessity of two local controllers: a controller for the force, and a controller for the current. These approaches increase the feasibility of a practical implementation given that its output is the current to apply to the $M R$ damper coil and they leave the saturation problem out.
When compared in the frequency domain with a passive suspension, the proposed controllers improved the comfort performance without affecting the suspension deflection and road holding. This improvement is in the order of 5-20 $\%$ for LPV-based and 10-20\% for FEB. When compared in time transient response, the FEB-Based controller accomplishes with the BS-6841 comfortable category, while the passive suspension and LPV-based control system are in the limit for little uncomfortable condition.

\section{REFERENCES}

Bolandhemmat, H., Clark, C.M., and Golnaraghi, F. (2009). Development of a Systematic and Practical Methodology for the Design of Vehicle Semi-active Suspension Control System. Veh. Syst. Dyn., 48(5), 567585.

Cartwright, K.V. (2007). Determining the Effective or RMS Voltage or Various Waveforms Without Calculus. The Tech. Interface, 8:1, 1-20.

Choi, S.B. and Sung, K.G. (2008). Vibration Control of Magnetorheological Damper System subjected to Parameter Variations. Int. J. Veh. Des., 46(1), 94-110.

Do, A.L., Sename, O., and Dugard, L. (2010). An LPV Control Approach for Semi-Active Suspension Control with Actuator Constraints. In Proc. of ACC 2010, 2010, Baltimore, $M D$.

Dong, X.M., Yu, M., Liao, C.R., and Chen, W.M. (2010). Comparative Research on Semi-Active Control Strategies for Magneto-Rheological Suspension. Nonlinear Dyn., 59, 433-453.

Griffin, M. J. (1996). Handbook of Human Vibration. Elsevier.

Ljung, L. (1999). System Identification : Theory for the user. Prentice Hall.

Lozoya-Santos, J., Sename, O., Dugard, L., MoralesMenendez, R., and Mendoza, R.R. (2010b). A LPV Quarter of Car with Semi-Active Suspension Model including Dynamic Input Saturation. In Proc. of IFAC SSSC 2010, Italy.

Poussot-Vassal, C., Sename, O., Dugard, L., Gáspár, P., Szabó, Z., and Bokor, J. (2008). A New Semi-active Suspension Control Strategy through LPV Technique. Control Eng. Practice, 16(12), 1519-1534.

Savaresi, S.M. and Spelta, C. (2009). A Single-Sensor Control Strategy for Semi-Active Suspensions. IEEE Trans. Control Syst. Technol., 17(1), 143-152.

Yu, M., Donga, X.M., Choi, S.B., and Liao, C.R. (2008). Human Simulated Intelligent Control of Vehicle Suspension System with MR Dampers. J. Sound Vibr., 319, $753-767$. 